\title{
Reflets
}

Revue ontaroise d'intervention sociale et communautaire

\section{Le recensement des outils de travail disponibles en français pour les professionnels oeuvrant dans le domaine de la santé : un rapport de la méthodologie utilisée par les physiothérapeutes}

\author{
Lynn Casimiro, Murielle Fortin, France Moisan et Louis E. Tremblay
}

Volume 8, numéro 1, printemps 2002

La réadaptation : son visage français en Ontario

URI : https://id.erudit.org/iderudit/026378ar

DOI : https://doi.org/10.7202/026378ar

Aller au sommaire du numéro

Éditeur(s)

Reflets : Revue ontaroise d'intervention sociale et communautaire

ISSN

1203-4576 (imprimé)

1712-8498 (numérique)

Découvrir la revue

Citer cet article

Casimiro, L., Fortin, M., Moisan, F. \& Tremblay, L. E. (2002). Le recensement des outils de travail disponibles en français pour les professionnels oeuvrant dans le domaine de la santé : un rapport de la méthodologie utilisée par les physiothérapeutes. Reflets, 8(1), 125-150. https://doi.org/10.7202/026378ar

Tous droits réservés (C) Reflets : Revue ontaroise d'intervention sociale et communautaire, 2002
Ce document est protégé par la loi sur le droit d'auteur. L'utilisation des services d'Érudit (y compris la reproduction) est assujettie à sa politique d'utilisation que vous pouvez consulter en ligne. 
Le recensement des outils de travail disponibles en français pour les professionnels oeuvrant dans le domaine de la santé :

\section{un rapport de la méthodologie utilisée par les physiothérapeutes}

Lynn Casimiro

Centre national de formation en santé

Regroupement des physiothérapeutes francophones de l'Ontario (RPFO).

Murielle Fortin

Physiothérapeute (clinique privée)

France Moisan

Physiothérapeute

Louis E. Tremblay ${ }^{1-2}$

École des sciences de la réadaptation (physiothérapie), l'Université d'Ottawa et président sortant du RPFO.

Au début des années 90, le Regroupement des physiothérapeutes francophones de l'Ontario (RPFO) consultait les physiothérapeutes ontariens parlant français pour connaitre les écueils et les difficultés associés à la pratique de la physiothérapie en milieu minoritaire francophone. Du sondage, il était clairement apparu que deux types d'outils manqueraient aux intervenants francophones pour leur permettre d'offrir des services optimaux 
à la population franco-ontarienne qu'ils desservaient. Le sondage révélait en effet l'absence d'outils en français facilitant les explications données aux clients au sujet, par exemple, des problèmes de santé ou des programmes d'exercices en milieux cliniques ou à domicile. Il manquait aussi aux physiothérapeutes et aux spécialistes de la réadaptation les outils d'évaluation en français pour répondre au virage de la pratique basée sur la mesure des résultats et les évidences scientifiques.

En 1995, le RPFO s'était donc donné deux objectifs fondamentaux. Le premier était de recenser les outils de traitement existant en français en Ontario et pouvant être partagés par les spécialistes de la réadaptation; le deuxième était de recenser les outils d'évaluation de langue française touchant le domaine de la physiothérapie.

Les bénévoles du RPFO, appuyés par le Regroupement des intervenantes et intervenants francophones en santé et en services sociaux de l'Ontario (RIFSSSO) le ministère des Ressources humaines du gouvernement canadien et l'Université d'Ottawa ont réussi à répertorier une gamme d'outils d'évaluation et de traitement utiles pour les physiothérapeutes oeuvrant dans les divers domaines de la physiothérapie. Le tout a été analysé, réorganisé et rassemblé.

Le présent article décrit la méthodologie que le RPFO a mise sur pied pour donner suite à ses objectifs. Quelques idées de base pour construire et proposer le protocole de recherche proviennent du regroupement des ergothérapeutes francophones de l'Ontario (REFO) qui poursuit le même objectif.

Cette dernière méthodologie peut être utile aux autres regroupements et organismes de professionnels intervenant en santé et en services sociaux puisqu' elle est adaptable à un groupe ou à un autre.

\section{Mandat}

L'étude pilote, sous forme de sondage, avait clairement démontré que les physiothérapeutes recherchaient deux types d'information. Le premier besoin était relié à l'avancement professionnel basé 
sur les preuves scientifiques. Il concernait donc un inventaire des outils scientifiques d'évaluation d'une condition ou d'un état, d'une déficience, d'une incapacité ou d'un handicap. L'intérêt pour ce genre de documents est à la hausse. En effet, la commande actuelle est de fonder sur des preuves la pratique de la physiothérapie qui sera alors définie comme le résultat de recherches orientées vers des décisions cliniques suite à une évaluation systématique de la condition du patient (Rosenberg et Donald 1995 : 1122-1126). Afin d'y arriver, il est nécessaire de dresser l'inventaire des outils d'évaluation. Edith Aston-McCrimmon (1999: 15-18) écrit :

Les physiothérapeutes cliniciens doivent pouvoir incorporer des mesures valables, fiables et réceptives des handicaps, des performances fonctionnelles et des invalidités :

1. Par le recours à des observations systématiques et la constitution de bases de données cliniques normalisées contenant des données permettant d'identifier les agents de prédiction des résultats d'un traitement afin d'évaluer plus exactement le pronostic client;

2. Par l'établissement d'instruments de mesure des résultats pour avoir un système de surveillance valable et systématique, afin de pouvoir juger de l'efficacité du traitement et d'évaluer la qualité du programme.

L'autre besoin consistait en la création d'une banque de données sur les outils de traitement. Dans un premier temps, les physiothérapeutes désiraient un inventaire des outils servant directement à leur travail de prescription, d'information et de communication auprès du client, de sa famille ou des aidants naturels. Dans cette catégorie se regroupent les logiciels d'exercices en français, les vidéos, les feuilles d'exercices spécifiques, les brochures reliées aux interventions en physiothérapie. Dans un deuxième temps, on a exprimé le souhait d'une liste des équipements de rééducation ayant une description et un mode de fonctionnement écrits en français et si possible, des références scientifiques quant à leur 
coût et à leur valeur réelle. Puis, dans un troisième temps, il s'est avéré intéressant et utile d'identifier les distributeurs d'équipements (logiciels, orthèses, etc.) capables de communiquer en français et d'offrir les services après-vente en français aux physiothérapeutes ou aux patients et à leur famille.

Le mandat du présent projet est donc double. Premièrement, il s'agit de faire l'inventaire des outils d'évaluation et deuxièmement celui des outils de traitement en langue française utilisés ou susceptibles de l'être par les physiothérapeutes, le personnel de soutien et les différentes institutions auprès de la clientèle qui les consulte. De plus, il fallait proposer différents canaux de diffusion des renseignements obtenus pour les rendre accessibles aux membres du RPFO et à tous les intéressés.

\section{Financement et ressources}

L'étude pilote avait été financée par les fonds internes du RPFO n'impliquant que de petites sommes autres que des salaires. Au printemps 1999, la présidente du RPFO et ses collaborateurs immédiats ont préparé et soumis une demande de fonds auprès $\mathrm{du}$ gouvernement canadien pour le programme Placement Carrière Été de Développement des ressources humaines Canada. La demande visait à défrayer le salaire au taux horaire minimum de trois étudiantes ou étudiants pour dix semaines de travail pendant l'été. Une des conditions à l'obtention de l'octroi était de développer un partenariat. Ce dernier a été établi entre le RPFO, le RIFSSSO et le programme de physiothérapie de l'Université d'Ottawa. Le gouvernement fédéral a subventionné le salaire d'une étudiante, soit le tiers de la demande. La subvention a été administrée par le RIFSSSO et l'Université d'Ottawa a fourni un local, un ordinateur, un téléphone, les photocopies, la papeterie. Le RPFO a défrayé les frais d'appels interurbains, de la poste et l'achat de certains documents utiles à l'étude.

Après l'annonce de l'octroi de la subvention par le gouvernement fédéral, trois chercheurs du RPFO gravitant aussi autour 
du programme de physiothérapie de l'Université d'Ottawa ont circonscrit le travail à accomplir et concilié les objectifs du RPFO et les règles de conduites dictées par les bailleurs de fonds. Nous avons recruté une étudiante autonome, maitrisant bien les deux langues officielles (beaucoup de milieux anglophones desservant des populations francophones devaient être contactés) parlées et écrites et possédant les habilités de base dans l'usage des ordinateurs. Périodiquement et à tour de rôle, les trois personnes-ressources rencontraient l'étudiante afin de lui fournir un encadrement et évaluer le progrès des travaux.

\section{Protocole de recherche}

L'hypothèse ou la question de recherche peut se résumer de la façon suivante : existe-t-il en réadaptation et/ou en physiothérapie, de la documentation pertinente en français, autre que les livres, que pourraient partager les physiothérapeutes ontariens oeuvrant auprès de la population francophone de la province?

L'étude proposée est dite non-expérimentale de type descriptif, qualitatif et quantitatif. L'approche au devis de recherche utilisée est l'enquête (Fortin et al. 1988: 74). Les modalités employées ont été d'abord des questionnaires administrés par courrier, par téléphone et parfois lors d'une entrevue puis, en dernier lieu, l'enquête bibliographique (périodiques, livres spécialisés, documents institutionnels, internet). L'échantillonnage fut le plus large possible, représentant un grand nombre d'établissements publics, privés et institutionnels de l'Ontario et, dans une moindre mesure, du Québec.

\section{Procédures}

Trois tâches spécifiques ont donc été délimitées pour mener à terme les objectifs de cette étude. Le recensement des outils 
d'évaluation (figure I), le recensement des outils de traitement (figure II) et l'édition ainsi que la publication des résultats (figure III).

Figure 1 - Inventaire des outils d'évaluation

\section{A. Inventaire des répertoires déjà complété d'autres organismes}

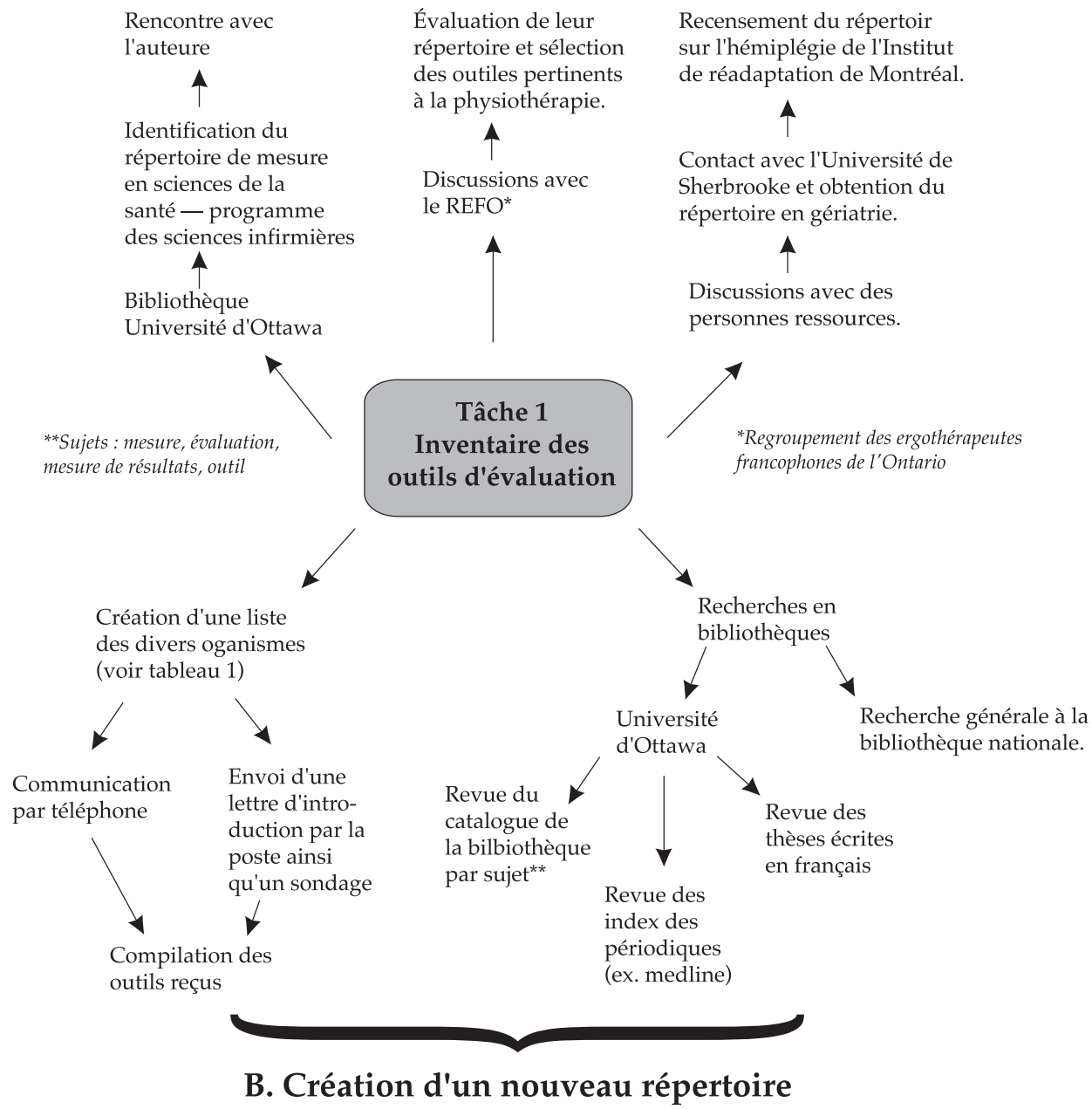


La figure illustre l'organigramme des étapes à suivre. Toute l'information recueillie a été compilée à l'aide d'une fiche informatisée (annexe III) et classée. La première étape (A) a consisté à faire le recensement du matériel existant. La partie $\mathrm{B}$ du tableau montre les étapes à suivre pour actualiser un répertoire des inventaires des outils d'évaluation propres à la physiothérapie. La liste des milieux contactés provient du site Internet du ministère de la Santé de l'Ontario et de la liste des placements cliniques de l'Université d'Ottawa.

Figure 2 - Inventaire des outils de traitement

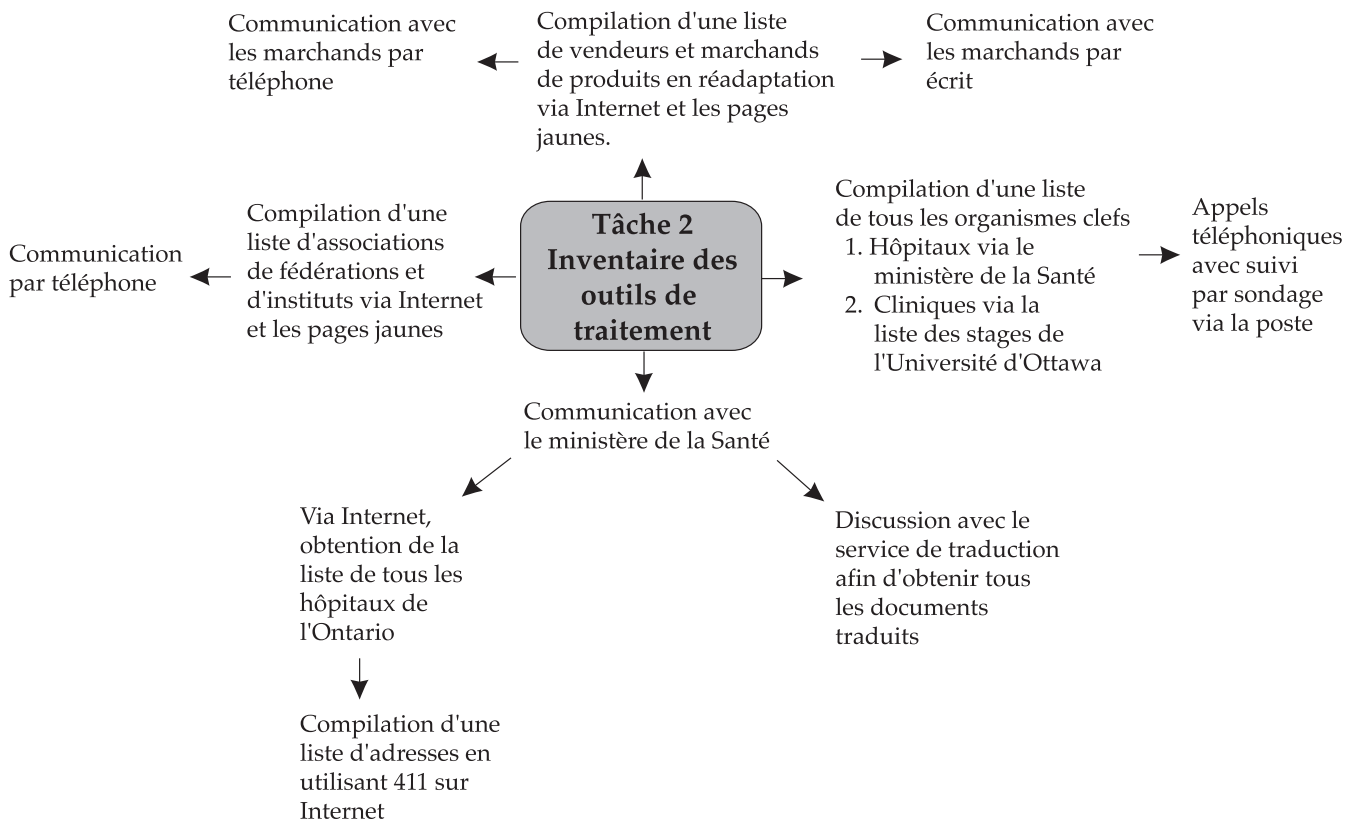

Quatre sources ont été identifiées pour l'inventaire des outils de traitement : le service de traduction du ministère de la Santé de l'Ontario; les centres hospitaliers et cliniques; les associations, fédérations et instituts (par ex., la Société de l'arthrite); des marchands de produits en réadaptation. La première étape consistait à dresser 
la liste des noms avec coordonnées. Ensuite, il a fallu communiquer avec les marchands et le ministère de la Santé. Pour les organismes, le contact initial a eu lieu lors du sondage, suivi de nombreux appels téléphoniques. Le sondage en question est le même que pour la tâche 1. Encore une fois, toute l'information recueillie a été compilée à l'aide d'une fiche informatisée (annexe 2) et a été classée dans un tableau sommaire.

Figure 3 - Publication et édition

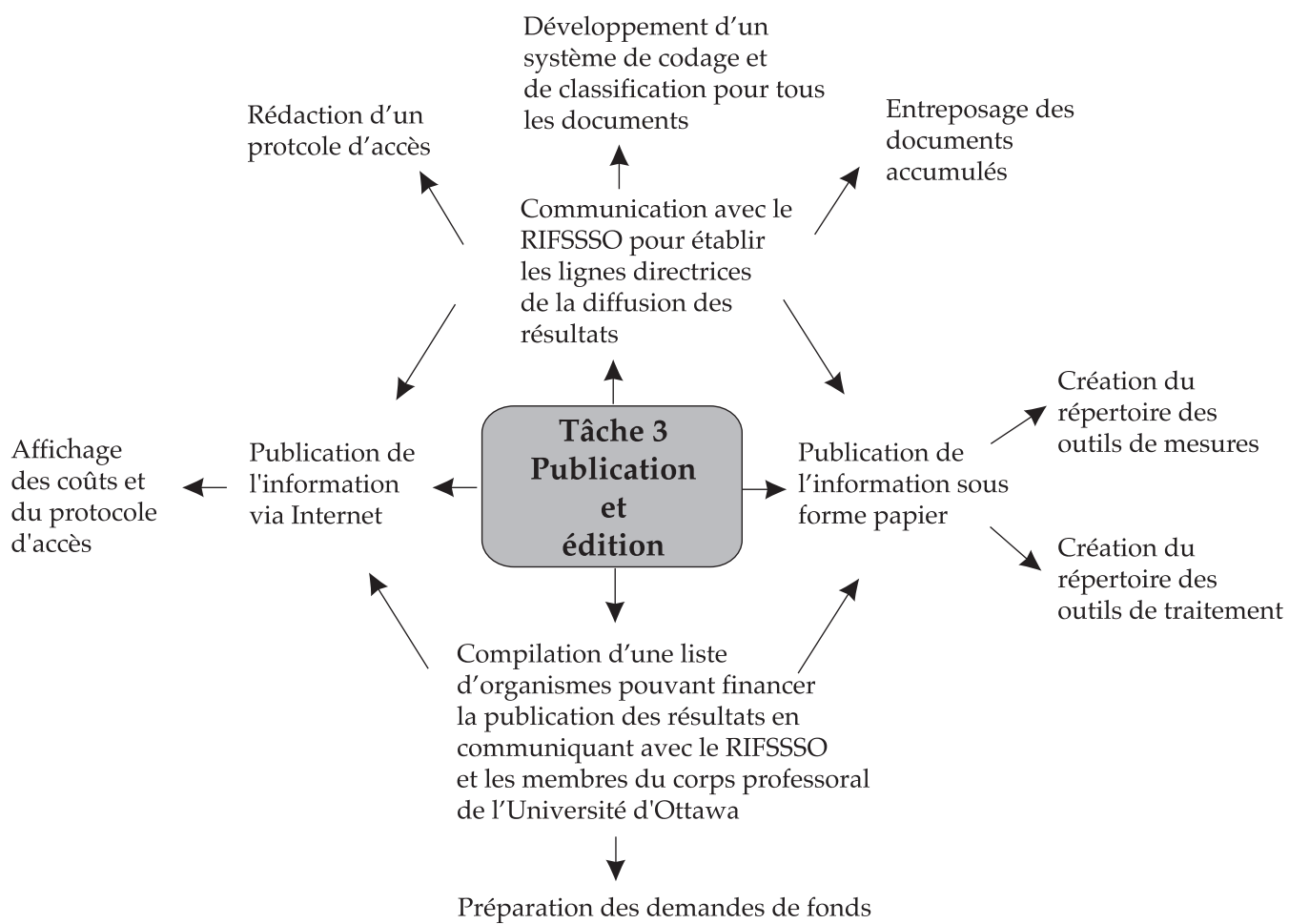

Avant de publier les résultats, il est nécessaire de déterminer un système de codage, de trouver un endroit d'entreposage et 
finalement, d'établir un protocole d'accès. Ce protocole doit déterminer qui aura droit à cette information, par quels processus et à quels coûts. Ces démarches ont été entreprises avec l'aide du RIFSSSO. Ensuite, diverses imprimeries ont été contactées pour déterminer les coûts de publication de deux répertoires, celui des outils de mesures et celui des outils de traitement. Les outils de traitement sont classés selon les catégories de services établies par l'Association canadienne de physiothérapie (ACP). Par la suite, les demandes de fonds auprès de divers organismes ont été complétées pour aider à défrayer les coûts d'imprimerie initiaux.

Tout d'abord, pour chacune des tâches, il a fallu dresser une liste de personnes-ressources (annexe I). Les pages jaunes de l'annuaire téléphonique et Internet ont été des plus utiles. Certaines pistes ont été retrouvées de façon fortuite, au hasard de conversations avec d'autres cliniciens ou chercheurs. Par exemple, la liste de stages de l'Université d'Ottawa, les connaissances de certains professeurs au sujet de répertoires existants ont toutes été prises en compte.

Ensuite, l'enquête ou le contact avec un milieu à l'aide d'une des modalités méthodologiques, le questionnaire par courrier (annexe II), les entrevues téléphoniques ou les rencontres en personne permettaient d'identifier si le milieu avait du matériel en français pertinent à l'une ou à l'autre des catégories d'information recherchée. Deux types de fiches standardisées ont été produits pour faire la cueillette des données. Pour la première tâche de l'étude, la fiche comprenait 17 éléments descriptifs décrivant et recensant les outils d'évaluation (annexe III). La fiche pour la deuxième tâche était liée aux outils de traitement (annexe IV). Elle comprenait 12 questions et commentaires.

Ce travail a dû être complété par des recherches bibliographiques. Cette étape visait plutôt les outils d'évaluation. Les deux ressources principales ont été la bibliothèque des Sciences de la santé de l'Université d'Ottawa et la Bibliothèque nationale. L'information recueillie a permis de trouver des documents d'appoint à ceux déjà répertoriés, de trouver des répertoires déjà publiés ainsi que des outils d'évaluation disponibles en français. 
La dernière étape pour les deux types d'outils était de réfléchir et de proposer une méthode de diffusion de l'information recueillie. Toute une série de consultations a été entreprise afin deconnaitre le meilleur véhicule pour disséminer les résultats de cette recherche, à savoir la création d'un catalogue ou d'un document informatisé sur Internet, ou d'une banque de données contenant les documents. Cependant, l'obtention des droits d'auteurs était un obstacle important à surmonter. Le RIFSSSO a pris la charge de cette tâche en communiquant avec les divers auteurs.

\section{Analyse des données}

\section{Conclusion}

L'analyse consistait à établir le pourcentage de répondants aux questionnaires des milieux échantillonnés. De plus, une analyse qualitative conservatrice a été faite afin de repérer les documents redondants et de les classer par catégories, soit outil d'évaluation ou outil de traitement. Chaque outil d'évaluation a ensuite été séparé selon les domaines du guide de l'Association canadienne de physiothérapie. En dernier lieu, une analyse descriptive a été faite et consignée dans une fiche validée. L'annexeV illustre cette fiche pour le test MAS (Motor assessement scale / L'échelle d'évaluation motrice). Pour ce qui est des outils de traitement, chacun a été trié, catalogué et inscrit dans un tableau sommaire (annexe VI).

Ce travail mené à terme par un petit groupe de chercheurs répondait à un désir exprimé par les physiothérapeutes de l'Ontario travaillant auprès de la population francophone. Quels ont été les fruits de ce labeur? Des centaines de documents ont 
été répertoriés et classés. Un site Web regroupant tous les outils de traitement en langue française sera accessible à tous les professionnels de la réadaptation au cours de l'année 2002. Un manuscrit présentant tous les outils d'évaluation répertoriés se retrouve chez les éditeurs au moment ou nous écrivons ces lignes. Ce travail entrepris depuis plusieurs années sur une base bénévole n'aurait pas avancé si rapidement en quelques mois n'eut été la subvention reçue du gouvernement canadien et du partenariat entre le RIFSSSO, le RPFO et l'Université d'Ottawa. Ce travail a aussi vu le jour grâce à l'autonomie, l'intelligence et le dévouement de l'étudiante chercheuse. C'est avec plaisir qu'elle a offert sa contribution à l'avancement des connaissances dans le domaine de la documentation francophone.

Pour réaliser une pareille tâche en si peu de temps, il faut donc de l'argent, du temps, de la persévérance, de l'organisation, du soutien et de la chance. De la chance parce qu'il faut que les personnes contactées soient disponibles. Précisément, ce projet s'est déroulé durant les mois d'été et les vacances étaient le plus grand obstacle. Un autre élément cause problème. Plusieurs établissements s'étaient engagés, sans résultat, à nous faire parvenir le matériel francophone dont ils disposaient. Même après quelques rappels, la situation ne s'est pas corrigée. Il n'y a pas eu d'autres problèmes majeurs si ce n'est les sautes d'humeur des ordinateurs.

En dépit de quelques exceptions, les milieux que nous avons contactés pour cette enquête nous ont aimablement consacré leur précieux temps. En effet, nous avons toujours été accueillis de façon courtoise. Nous avons fait miroiter que le travail accompli dans le présent projet aura sans doute des retombées positives pour leur milieu et leur pratique.

Il nous est facile de répondre positivement à la question de recherche. Il y a donc, de façon significative, une documentation francophone en usage dans les différents milieux de la physiothérapie en Ontario. De plus, plusieurs outils d'évaluation en français sont utilisés par ces derniers.

Nous croyons que la description du projet réalisé par le RPFO démontre bien que la démarche est transférable aux autres 
organismes dans leur recherche du matériel en français que souhaitent les intervenantes et intervenants en services sociaux et de santé pour une pratique efficace et respectueuse de leur clientèle.

\section{Bibliographie}

ASTON-McCrimmon, E. (1999). "La profession de physiothérapeute en l'an 2000 et au-delà », Physio-Québec, 23:4, 15-18.

COLE, B., E. FINCH, C. GOWLANd et N. MAYO (1995). «Instruments de mesure des résultats en réadaptation ", dans J. Bosmajion, éd. Santé Canada et l'Association canadienne de physiothérapie, 220.

FORTIN, M.F., M.E.TAGGART, S. KEROUAC et S. NORMAND (1988). Introduction à la recherche, Montréal, Décarie.

FORTIN, F., G. COUTU-WAKULCZYK et F. FILION (1996). Répertoire d'instruments de mesure : Application à la santé et aux domaines connexes, Rapport intérimaire, Université d'Ottawa, Sciences infirmières, 73-76.

HÉBERT,. R., G. BRAVO et L.VOYER (1993). Répertoire des instruments de mesure en langue française pour la recherche gérontologique et gériatrique, Sherbrooke, Centre de recherche en gérontologie et gériatrie.

LE REGROUPEMENT des ergothérapeutes francophones de l'Ontario (1998). Le recensement des outils de travail en ergothérapie. Document non publié.

LE REGROUPEMENT des physiothérapeutes francophones de l'Ontario (1997). «Sondage de l'inventaire des documents français ", Le Courant, 4, 1-11.

ROSENBERG, W. et A. DONALD (1995). «Evidence based medecin : an approach to clinical problem-solving ", British Medical Journal, 310, 1122-1126.

\section{Notes}

1. Veuillez adresser toutte correspondance à Dr. Louis E. Tremblay, Programme de physiothérapie, École des sciences de la santé, Université d'Ottawa, 451 rue Smyth, Ottawa (Ontario), K1H 8M5, téléphone (613) 562-5800, poste 8455, télécopieur : (613) 562-5428, courriel : 1trembl@aix1.uottawa.ca.

2. Nous reconnaissons la généreuse participation du ministère des Ressources humaines du Canada, du RIFSSSO, du RPFO, de la Fondation franco-ontarienne et de l'Université d'Ottawa. 


\section{Annexe I}

\section{Liste des milieux et des écrits consultés pour faire l'inventaire des instruments de mesure en réadaptation / physiothérapie}

\section{Enquête par courrier, téléphone ou entrevue}

Regroupement des ergothérapeutes francophones de l'Ontario (REFO) .................. Toronto

Regroupement des physiothérapeutes francophone de l'Ontario (RPFO) ............... Toronto

Ordre des physiothérapeutes de $\mathrm{l}^{\prime}$ Ontario ................................................................... Toronto

Ordre des physiothérapeutes du Québec .................................................................. Montréal

Association ontarienne de physiothérapie ................................................................... Toronto

Association canadienne de physiothérapie ............................................................... Toronto

Association canadienne pour la recherche en réadaptation ...................................... Edmonton

Fédération des physiothérapeutes du Québec ........................................................... Montréal

Université d'Ottawa (physiothérapie, ergothérapie) ................................................... Ottawa

Université de Montréal (physiothérapie) ................................................................... Montréal

Université Laval (physiothérapie) .............................................................................. Québec

Université de Sherbrooke (centre de recherche en géronto-gériatrie) ...................... Sherbrooke

Université McGill (physiotherapy, exercise science) .................................................... Montréal

Université Concordia (exercise science) …................................................................. Montréal

Université McMaster (physiotherapy) ......................................................................... Hamilton

Institut de réadaptation de Montréal ........................................................................... Montréal

Institut de réadaptation en déficience physique de Québec ...................................... Québec

Centre de réadaptation Lucie Bruneau ........................................................................ Montréal

Centre hospitalier St-Augustin ................................................................................ Québec

Centre hospitalier des Vallées de l'Outaouais ........................................................... Hull-Gatineau

Hôpital d'Ottawa (Campus Général) .......................................................................... Ottawa

Hôpital St-Justine ...................................................................................................... Montréal

Hôpital Royal Victoria ................................................................................................ Montréal

Hôpital Montfort ............................................................................................................... Ottawa

Société canadienne de physiologie de l'exercice ........................................................ Ottawa

Les fondations canadiennes de certaines maladies (pulmonaires,...) ....................... Ottawa 


\section{Annexe 1 - suite}

\section{Enquête bibliographique}

Répertoire des instruments de mesure en ergothérapie (REFO) ................................ Ottawa

Système de recherche bibliographique Polaris - Medline

Ottawa

Index d'article de revue en langue française (REPÈRE), système

Orbis de l'Université d'Ottawa

Ottawa

Index des thèses de la Bibliothèque Nationale du Canada Ottawa

Références des articles scientifiques répertoriés Ottawa

Répertoire des instruments de mesure en langue française pour la recherche gérontologique et gériatrique Sherbrooke

Répertoire d'instruments de mesure : Application à la santé et aux domaines connexes, Rapport intérimaire Ottawa

Instruments de mesure des résultats en réadaptation publié par l'Association canadienne de physiothérapie. Toronto 


\section{Annexe II}

\section{Exemple du questionnaire utilisé SONDAGE TÉLÉPHONIQUE PRÉLIMINAIRE}

\begin{tabular}{|c|c|c|c|c|}
\hline & OUI & NON & $\begin{array}{l}\text { Peut- } \\
\text { être }\end{array}$ & $\begin{array}{l}\text { Commentaires/ } \\
\text { Suggestions }\end{array}$ \\
\hline \multicolumn{5}{|l|}{$\begin{array}{l}\text { Est-ce que votre institution fait usage } \\
\text { d'instruments de mesure francophones? }\end{array}$} \\
\hline \multicolumn{5}{|l|}{$\begin{array}{l}\text { 1. Instruments de mesure de l'activité } \\
\text { motrice et de la capacité fonctionnelle } \\
\text { chez l'adulte? }\end{array}$} \\
\hline \multicolumn{5}{|l|}{$\begin{array}{l}\text { 2. Instruments de mesure de la douleur } \\
\text { et des problèmes vertébraux? }\end{array}$} \\
\hline \multicolumn{5}{|l|}{$\begin{array}{l}\text { 3. Instruments de mesure des paramètres } \\
\text { cardio-pulmonaires }\end{array}$} \\
\hline \multicolumn{5}{|l|}{$\begin{array}{l}\text { 4. Instruments de mesure du } \\
\text { développement de l'enfant? }\end{array}$} \\
\hline \multicolumn{5}{|l|}{$\begin{array}{l}\text { Est-ce que votre institution possède de la } \\
\text { documentation francophone destinée au } \\
\text { patient en physiothérapie? (ex. : programme } \\
\text { d'exercices, information sur pathologie,...) }\end{array}$} \\
\hline \multicolumn{5}{|l|}{$\begin{array}{l}\text { Est-ce que votre institution serait prête à } \\
\text { rendre ces outils de travail disponibles au } \\
\text { centre de documentation géré par le } \\
\text { RIFSSSO? }\end{array}$} \\
\hline \multicolumn{5}{|l|}{$\begin{array}{l}\text { Si oui, quelles sont les procédures à suivre, } \\
\text { qu'elle est la personne contact, ect... }\end{array}$} \\
\hline \multicolumn{5}{|l|}{$\begin{array}{l}\text { Si non, pour fin de sondage, pourriez- } \\
\text { vous nous dire pourquoi vous ne désirez } \\
\text { pas participer? }\end{array}$} \\
\hline $\begin{array}{l}\text { Et est-ce que votre institution serait prête à } \\
\text { fournir une liste des titres de documents } \\
\text { francophones qu'elle possède par fin } \\
\text { d'inventaire? }\end{array}$ & & & & \\
\hline
\end{tabular}




\begin{tabular}{|l|l|l|l|l|}
\hline & OUI & NON & $\begin{array}{c}\text { Peut- } \\
\text { être }\end{array}$ & $\begin{array}{c}\text { Commentaires/ } \\
\text { Suggestions }\end{array}$ \\
\hline $\begin{array}{l}\text { Pour terminer, est-ce que votre institution } \\
\text { aimerait recevoir de l'information sur le } \\
\text { progrès du dossier? }\end{array}$ & & & & \\
\hline $\begin{array}{l}\text { Est-ce que votre institution souhaite de } \\
\text { plus amples renseigments au sujet de } \\
\text { notre regroupement (RPFO)? }\end{array}$ & & & & \\
\hline
\end{tabular}




\section{Annexe III}

\section{Exemple de la fiche pour outil d'évaluation}

Auteurs :

Clientèle :

Utilité clinique :

Composé de :

Durée d'administration :

Par qui :

Environnement requis :

Matériel requis :

Items de l'évaluation :

Méthode :

Interprétation des résultats :

Caractéristiques psychométriques :

Formation requise :

Où le consulter : 


\section{Annexe 3 - suite}

Où se le procurer :

Références : 


\section{Annexe IV}

Exemple de la fiche pour outil de traitement

\begin{tabular}{|l|l|l|l|}
\hline Date & Titre & $\begin{array}{c}\text { Auteur } \\
\text { Institution }\end{array}$ & Contenu \\
\hline & & & \\
\hline & & & \\
\hline & & & \\
\hline & & & \\
\hline
\end{tabular}




\section{Annexe V}

\section{Illustration de l'utilisation de la fiche d'inscription des outils d'évaluation pour le test \\ Profil de mobilité fonctionnelle en physiothérapie (PMFP)}

Auteurs :

Platt W., Bell B., Kozak J.

Pavillon Élisabeth Bruyère

Hôpital des Soeurs de la Charité d'Ottawa

Clientèle :

Clients recevant des soins de longue durée ou aiguë.

Utilité clinique :

Permet d'évaluer et de mesurer la mobilité fonctionnelle d'un adulte en soin de longue durée ou aiguë. Permet d'établir des buts de traitements.

Composé de :

Formulaire d'une page du PMFP

Durée d'administration : Environ 10 à 15 minutes

Par qui :

Une physiothérapeute afin d'obtenir l'uniformité des qualifications au niveau des évaluateurs.

Environnement requis : L'environnement requis doit être optimal. Par exemple, si le client peut seulement se rouler dans une direction, le lit peut être changer de place afin de promouvoir la capacité fonctionnelle. 


\section{Annexe 5-suite}

Matériel requis :

Le formulaire PFMP, un escaliers de $18,5 \mathrm{~cm}$ de haut avec rampe et une rampe possédant un angle de trois pourcent.

Items de l'évaluation: L'évaluation mesure objectivement neuf domaines : la mobilité au lit, le passage de la position couchée à la position assise, l'équilibre en position assise, le passage de la position assise à la position debout, l'équilibre en position debout, les transfert, le déplacement en chaise roulante, la marche et les escaliers.

Méthode :

L'évaluation initiale doit être faite dans les deux premières semaines suivant l'admission. Le formulaire est rempli suite aux observations faites par une physiothérapeute.

Interprétation des résultats :

Chaque domaine est évalué à l'aide d'une échelle de sept points. Un score de 7 indique une indépendance complète tandis qu'un score de 1 indique une dépendance complète. Les points obtenus dans chaque domaine sont ensuite additionnés enfin d'obtenir une image du statut de l'ensemble de la mobilité fonctionnelle du client. Le score maximal est de 63 tandis que le score minimal est de 7 . 


\section{Annexe 5 - suite}

\section{Caractéristiques psychométriques :}

L'outil d'évaluation n'a été validé qu'en langue anglaise.

1. Pour une population en soins longue durée :

Fidélité inter-item : $\quad$ La corrélation (Pearson Product Moment) entre les items variaient de 0,47 à 0,93 . Ceci nous indique une bonne variété entre les items, il y a présence de moins de redondance entre les items.

Consistence interne : $\quad 0,96$; excellente

Fidélité inter-examinateur: Pour l'ensemble de l'évaluation : 0,95;

excellente. Pour chaque domaine : 0,47 à 0,93; bonne.

Fidélité intra-examinateur : Les examinateurs n'ont pas changé leurs scores dans le temps. Les scores sont demeurés stable dans le temps.

Sensibilité :

Le PMFP est sensible au paramètre clinique externe, au statut du client lors de son admission, au changement de la mobilité fonctionnelle et au lieu ou le client se dirige après son congé de l'hôpital

\section{Pour client en milieu aiguë :}

Fidélité intra-examinateur :

Fidélité inter-examinateur :

Consistence interne :
0,99 ; excellente

0,97 ; excellente

0,99 ; excellente 


\section{Annexe 5 - suite}

Validité de construction : Démontre que le PMFP étudie seulement un facteur principal : la capacité.

Formation requise :

Non-mentionnée

Où le consultater :

Brenda Bell

Physiothérapie / Complex Continuing Care

Hôpital des Soeurs de la Charité d'Ottawa

Pavillon Elisabeth Bruyère

43 , rue Bruyère, Ottawa ON K1N $5 \mathrm{C} 8$

(613) 562-0050, poste 1205

Où de le procurer : $\quad$ Brenda Bell

Physiothérapie / Complex Continuing Care Hôpital des Soeurs de la Charité d'Ottawa

Pavillon Elisabeth Bruyère

43 , rue Bruyère, Ottawa ON K1N 5C8

(613) 562-0050, poste 1205

L'outil de mesure est gratuit mais il est nécessaire de mentionner les Soeurs de la Charité lors de l'utilisation de l'outil.

\section{Références}

Platt W., Bell B., Kozak J. (1998) Physiotherapy Functional Mobility Profile, A Tool for Measuring Functional Outcome in Chronic Care Clients. Physiotherapy Canada, 50 (1) $47-52,74$.

Brosseau L., Laferrière L., Couroux N., Marion M., Thériault J. Intra and Inter-Rater(s) Reliability and Factorial Validity Studies of the "Physiotherapy Functional Mobility Profile (PFMP)" Conducted with Inpatient Subjects Treated in an Acute Care Hospital. Article sous presse pour le journal Physiotherapy: Theory and Practice. 


\section{Exemple du tableau sommaire des outils de traitement Inventaire des documents de l'hôpital Glengarry Memorial}

\begin{tabular}{|c|c|c|c|}
\hline Date & Titre & $\begin{array}{c}\text { Auteur } \\
\text { Institution }\end{array}$ & Contenu \\
\hline \multirow[t]{4}{*}{1995} & $\begin{array}{l}\text { Exercices - remplacement } \\
\text { total du genou }\end{array}$ & Glengarry Mem. Hosp. & $\begin{array}{l}\text { Neuf exercices avec schémas } \\
\text { à faire suite à un remplacement } \\
\text { total du genou. (1 page) }\end{array}$ \\
\hline & Exercices de flexion lombaire & Glengarry Mem. Hosp. & $\begin{array}{l}\text { Neuf exercices de flexion } \\
\text { lombaire pour gens souffrant } \\
\text { d'ostéoporose. (1 page) }\end{array}$ \\
\hline & Bursite, tendinite de l'épaule & SYNTEX & $\begin{array}{l}\text { Le document comprend des } \\
\text { schémas, la description de la } \\
\text { blessure, les facteurs } \\
\text { prédisposants, le traitement, } \\
\text { les sports et les exercices. } \\
\text { (2 pages) }\end{array}$ \\
\hline & $\begin{array}{l}\text { Épicondylite des joueurs } \\
\text { de tennis/golf }\end{array}$ & SYNTEX & $\begin{array}{l}\text { Le document comprend des } \\
\text { schémas, la description de la } \\
\text { blessure, les facteurs } \\
\text { prédisposants, le traitement, les } \\
\text { sports et les exercices. (2 pages) }\end{array}$ \\
\hline 1990 & Exercices pour épicondylite & Glengarry Mem. Hosp. & $\begin{array}{l}\text { Exercices et progression pour } \\
\text { épicondylite afin d'augmenter } \\
\text { la flexibilité des muscles et } \\
\text { d'augmenter la force } \\
\text { musculaire. Le document } \\
\text { comprend également des } \\
\text { exercices isotoniques. ( } 3 \text { pages) }\end{array}$ \\
\hline \multirow[t]{2}{*}{1990} & $\begin{array}{l}\text { Exercices pour une fracture } \\
\text { de colles }\end{array}$ & Glengarry Mem. Hosp. & $\begin{array}{l}\text { Exercices pour le poignet, } \\
\text { le coude, la main et l'épaule } \\
\text { lors d'une fracture de colles. } \\
\text { ( } 2 \text { pages) }\end{array}$ \\
\hline & Exercices post-nataux & Glengarry Mem. Hosp. & $\begin{array}{l}\text { Exercices post-nataux pour } \\
\text { améliorer le tonus musculaire } \\
\text { de l'abdomen, pour améliorer } \\
\text { la posture et pour augmenter } \\
\text { l'habileté à relaxer. (1 page) }\end{array}$ \\
\hline
\end{tabular}




\section{Annexe VI - suite}

\begin{tabular}{|c|l|l|l|}
\hline Date & \multicolumn{1}{|c|}{ Titre } & \multicolumn{1}{|c|}{$\begin{array}{c}\text { Auteur } \\
\text { Institution }\end{array}$} & \multicolumn{1}{c|}{ Contenu } \\
\hline \multirow{2}{*}{1993} & Sans titre & Glengarry Mem. Hosp. & $\begin{array}{l}\text { Brève description du principe } \\
\text { de protection, repos, glace, } \\
\text { compression et élévation. } \\
\text { Comprend également une } \\
\text { courte description de choses à } \\
\text { ne pas faire et à faire suite à } \\
\text { une blessure. (1 page) }\end{array}$ \\
\hline $\begin{array}{l}\text { Directives pour marcher } \\
\text { avec des béquilles }\end{array}$ & Glengarry Mem. Hosp. & $\begin{array}{l}\text { Comment utiliser des } \\
\text { béquilles avec appui partiel, } \\
\text { sans appui et comment monter } \\
\text { et descendre les escaliers avec } \\
\text { des béquilles. }\end{array}$ \\
\hline
\end{tabular}

